\title{
Analysis of shielding layers in HTS cable taking account of spiral structure
}

\section{$\operatorname{AUTHOR}(\mathrm{S})$ :}

Nakamura, T; Kanzaki, H; Higashikawa, K; Hoshino, T; Muta, I

\section{CITATION:}

Nakamura, T ...[et al]. Analysis of shielding layers in HTS cable taking account of spiral structure. IEEE TRANSACTIONS ON APPLIED SUPERCONDUCTIVITY 2005, 15(2): 1747-1750

\section{ISSUE DATE:}

2005-06

URL:

http://hdl.handle.net/2433/39959

\section{RIGHT:}

(c)2005 IEEE. Personal use of this material is permitted. However, permission to reprint/republish this material for advertising or promotional purposes or for creating new collective works for resale or redistribution to servers or lists, or to reuse any copyrighted component of this work in other works must be obtained from the IEEE. 


\title{
Analysis of Shielding Layers in HTS Cable Taking Account of Spiral Structure
}

\author{
Taketsune Nakamura, Haruyuki Kanzaki, Kohei Higashikawa, Tsutomu Hoshino, and Itsuya Muta
}

\begin{abstract}
Analysis code for shielding layers of HTS cable, which was taken account of spiral structure of $\mathrm{Bi}-2223 / \mathrm{Ag}$ tape conductors, was developed based on finite element method. The $66 \mathrm{kV}$ class HTS cable, which was fabricated and successfully tested in cooperation of Tokyo Electric Power Co. (TEPCO) and Sumitomo Electric Industries, Ltd. (SEI), was subjected in this study. Electric field vs. current density property was expressed based on percolation depinning model (Yamafuji-Kiss model). Multifilamentary structure of the tapes and spiral structure of the conductors were directly modeled in order to analyze precisely. Systematic analysis results were presented and discussed.
\end{abstract}

Index Terms-Finite element method, HTS cable, multifilamentary structure, percolation depinning model, spiral structure.

\section{INTRODUCTION}

$\mathbf{R}$ ECENT advances in performance improvement as well as long length fabrication technology of $\mathrm{Bi}-2223 / \mathrm{Ag}$ tape conductors have continuously stimulated the high- $T_{\mathrm{c}}$ superconducting (HTS) power applications. As one of such most promising candidates for commercialization is HTS power transmission cable, and have intensively and extensively been developed in the world [1]-[5]. Especially, Tokyo Electric Power Co. (TEPCO) and Sumitomo Electric Industries, Ltd. (SEI) in Japan had already fabricated and successfully tested a 100 m class HTS cable system [2], [3]. As a next step, a 500 $m$ length HTS cable has been installed and under testing as Super-ACE national project [6].

In order to realize such HTS cable system, their performances have to be estimated precisely. Current transport properties in HTS, however, vary complicatedly and nonlinearly depending on the operational temperature, experienced magnetic field, its applied direction (magnetic anisotropy) and so forth. Furthermore, HTS cable generally consists of Bi-2223/Ag tapes that has multifilamentary structure, and such tapes are wound spirally around the former. Therefore, the effect of such complicated structures upon the cable performance has also to be investigated.

In this paper, we developed the analysis code for magnetic shielding layers of HTS cable based on 3-dimensional finite element method (3D-FEM). These HTS shielding layers are crucial

Manuscript received October 4, 2004. This work was supported in part by 21st Century COE Program (no. 14213201) from The Ministry of Education, Culture, Sports, Science and Technology, Japan.

T. Nakamura, H. Kanzaki, K. Higashikawa, and T. Hoshino are with the Kyoto University, Kyoto-Daigaku Katsura 1, Kyoto 615-8510, Japan (e-mail: tk_naka@kuee.kyoto-u.ac.jp; kohei@asl.kuee.kyoto-u.ac.jp; hoshino@asl.kuee.kyoto-u.ac.jp).

I. Muta is with the Saga University, Honjo-Cho 1, Saga 840-8502, Japan (e-mail: muta128@bb.excite.co.jp).

Digital Object Identifier 10.1109/TASC.2005.849272 for avoidance of leakage magnetic field induced from the conducting layers. The current shielding performance is evaluated for arbitrary temperature, magnetic field and its applied direction by using analytical expressions of current transport properties. The analysis results are to be presented for different conducting currents and operating temperatures.

\section{ANALYSIS METHOD}

\section{A. J-E Constitutive Relation}

In this study, we consider the $J-E$ constitutive relation based on percolation depinning model, which has been proposed by Yamafuji and Kiss [7], and then formulated by Kiss et al. [8]. Further, we have extended the expression in order to describe the magnetic anisotropy [9]. The detailed expressions are shown as follows:

$$
\begin{aligned}
& E(J, T, B, \varphi)= \\
& \frac{\rho_{\mathrm{ff}}}{m+1} J\left(\frac{J}{J_{0}(T, B, \varphi)}\right)^{m} \\
& \quad \cdot\left(1-\frac{J_{\mathrm{cm}}(T, B, \varphi)}{J}\right)^{m+1} ; \quad B \leq B \mathrm{~g} \\
& \frac{\rho_{\mathrm{ff}}}{m+1}\left|J_{\mathrm{cm}}(T, B, \varphi)\right|\left(\frac{\left|J_{\mathrm{cm}}(T, B, \varphi)\right|}{J_{0}(T, B, \varphi)}\right)^{m} \\
& \quad \cdot\left[\left(1+\frac{J}{\left|J_{\mathrm{cm}}(T, B, \varphi)\right|}\right)^{m+1}-1\right] ; \quad B>B \mathrm{~g}
\end{aligned}
$$

where, $\rho_{\mathrm{ff}}$ and $m$ denote the uniform flux-flow resistivity and the parameter describing the shape of the local critical current density distribution. $\varphi$ is defined as the angle between the applied direction of external magnetic field and the normal axis with respect to the tape's surface. $J_{\mathrm{cm}}$ and $J_{0}$, respectively, mean the minimum value and width of the local critical current distribution, and expressed as follows:

$$
\begin{aligned}
J_{\mathrm{cm}}(T, B, \varphi) & =J_{\mathrm{B}}\left|1-\frac{B}{B_{\mathrm{g}}(T, \varphi)}\right|^{2 \nu} \\
J_{0}(T, B, \varphi) & =A_{0} T^{\xi} B_{\mathrm{eq}}(B, \varphi)^{\eta}
\end{aligned}
$$

where, $J_{\mathrm{B}}$ and $A_{0}$ are the coefficients, and $\nu, \xi$ and $\eta$ are indices. Further, $B_{\mathrm{g}}$ and $B_{\mathrm{eq}}$ are so-called glass-liquid transition magnetic flux density and the equivalently perpendicular magnetic flux density, respectively, and expressed as,

$$
\begin{aligned}
B_{\mathrm{eq}}(B, \varphi) & =B\left(\cos ^{2} \varphi+\gamma^{-2} \sin ^{2} \varphi\right)^{\frac{1}{2}} \\
B_{\mathrm{g}}(T, \varphi) & =\frac{B_{\mathrm{g}}\left(0 \mathrm{~K}, 0^{\circ}\right)}{B_{\mathrm{eq}}(B, \varphi)} B\left(1-\frac{T}{T_{\mathrm{c}}}\right)^{\alpha}
\end{aligned}
$$




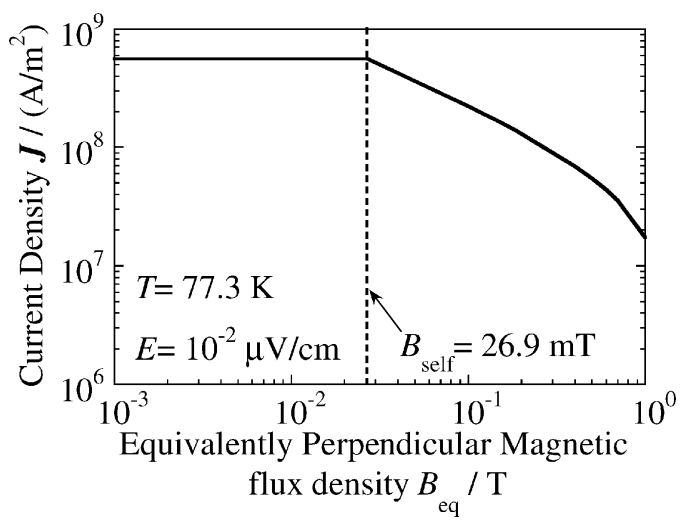

Fig. 1. Current density $J$ of Bi-2223/Ag tape defined at electric field criterion with $10^{-2} \mu \mathrm{V} / \mathrm{cm}$ and its threshold.

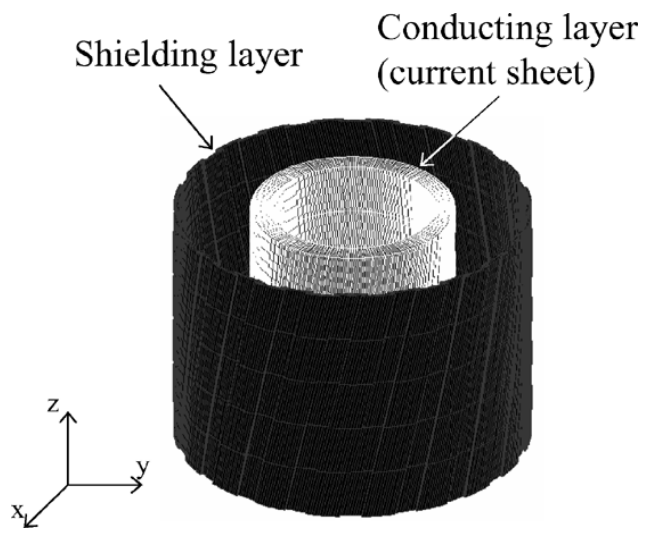

Fig. 2. Schematic diagram of analysis model for shielding layer in HTS cable. Conducting layer is regarded as a current sheet.

where, $\gamma\left(=B_{\mathrm{g}}\left(T, \varphi=90^{\circ}\right) / B_{\mathrm{g}}\left(T, \varphi=0^{\circ}\right)\right)$ denotes the anisotropy factor, and $T_{\mathrm{c}}$ is critical temperature.

We also determine the threshold values for the current density, $J[10]$. That is, when $B_{\mathrm{eq}}$ at one mesh in Bi-2223 filaments is less than the self-field, $B_{\text {self }}(=26.9 \mathrm{mT})$, the current density at this mesh is set to the constant value calculated by using (1)-(6) with $B_{\text {self }}$. Here $B_{\text {self }}$ is defined by the field at which $J-E$ curve calculated using (1)-(6) equals one obtained from the measurement at self-field. For instance, Fig. 1 shows the double logarithmic plots of $J$ defined by the electric field criterion at $10^{-2} \mu \mathrm{V} / \mathrm{cm}$, as a function of $B_{\text {eq }}$. When $B_{\text {eq }}>B_{\text {self }}$, the current density is determined by (1)-(6). On the other hand, $J$ is set to be a constant value in case of $B_{\text {eq }}<B_{\text {self }}$.

\section{B. Analysis Model}

Fig. 2 shows the analysis model of HTS cable. The shielding layers are only considered for the analysis, and the conducting layers are treated as current sheets (total conducting current: $I$ ). The number of the shielding layers is 2 , and each of them consists of 20 tape conductors surrounding the conducting layers. The spiral pitch length is $500 \mathrm{~mm}$. By using the symmetry of the electromagnetic field, the length along $z$-axis with $1 / 20$ of the spiral pitch is only modeled, and then the upper- as well as lower-most surface is set to be parallel periodic boundary condition. In other words, we assume the infinitely long length of the HTS cable, and the shielding current is considered to be only

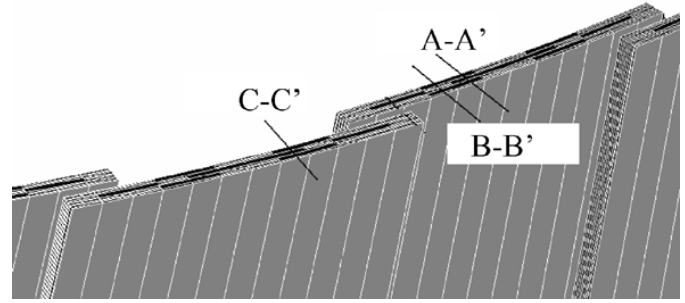

Fig. 3. Enlarged cross section of the analysis model. Lines A-A', B-B' and $\mathrm{C}-\mathrm{C}^{\prime}$ are for the calculation of magnetic field and current density distribution.

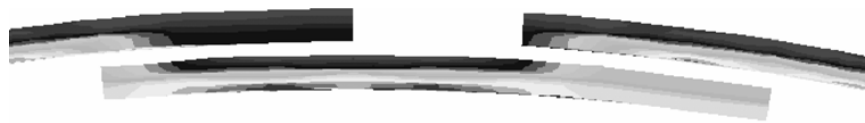

(a)

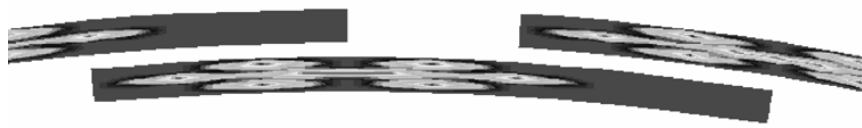

(b)

Fig. 4. Example of analysis results $(I=3.8 \mathrm{kA}, T=77.3 \mathrm{~K})$. (a) Contour plot of magnetic flux density. (b) Contour plot of current density.

induced by the magnetic field generated by the conducting current, I. Fig. 3 also shows the enlarged cross section of the analysis model. Each of Bi-2223/Ag multifilamentary tapes consists of 7 filaments, and the mesh number for each are $4 \times 2$. Lines $\mathrm{A}-\mathrm{A}^{\prime}, \mathrm{B}-\mathrm{B}^{\prime}$ and $\mathrm{C}-\mathrm{C}^{\prime}$ in the figure are for showing the radial distribution of the electromagnetic field along these lines.

As is already shown, $J-E$ constitutive relation in this analysis includes the magnetic anisotropy. Therefore, the applied direction of the magnetic field with respect to the surface of each mesh should be determined in Bi-2223 filaments. In order to calculate the applied angle, $\varphi$, the following expression can easily be derived.

$$
\varphi=\tan ^{-1}\left[\frac{\frac{l_{\mathrm{p}}}{2 \pi}\left(B_{\mathrm{x}} y-B_{\mathrm{y}} x\right)+B_{\mathrm{z}} r^{2}}{\left(B_{\mathrm{x}} x-B_{\mathrm{y}} y\right) \cdot \sqrt{\left(\frac{l_{\mathrm{p}}}{2 \pi}\right)^{2}+r^{2}}}\right]
$$

where, $B_{\mathrm{i}}(\mathrm{i}=\mathrm{x}, \mathrm{y}, \mathrm{z})$ denotes the i-axis component of the magnetic flux density. Parameters $l_{\mathrm{p}}(=500 \mathrm{~mm})$ and $r$ mean, respectively, the spiral pitch and mean radius of each mesh in the shielding layers.

\section{RESULTS AND DISCUSSION}

\section{A. Shielding Performance for Different Conducting Currents $(\mathrm{T}=77.3 \mathrm{~K})$}

Fig. 4 shows the contour plots of, respectively, (a) magnetic flux density and (b) current density obtained for $I=3.8 \mathrm{kA}$ and $T=77.3 \mathrm{~K}$. We can see that the shielding current is concentrated in the Bi-2223 filaments at this operating condition (Fig. 4(b)). By using these results, the distribution of electromagnetic field is calculated. Fig. 5 shows the distributions of (a) magnetic flux density and (b) current density along A- $\mathrm{A}^{\prime}$,

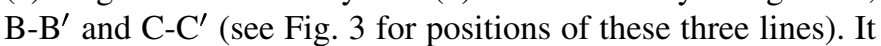
can be seen that the magnetic flux density is almost shielded by only one shielding layer, i.e., each of lines passes through 


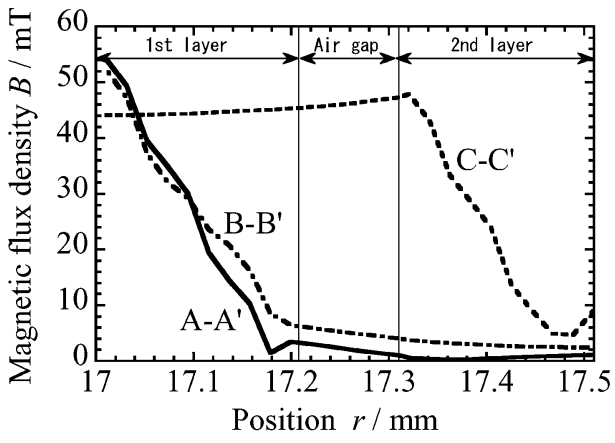

(a)

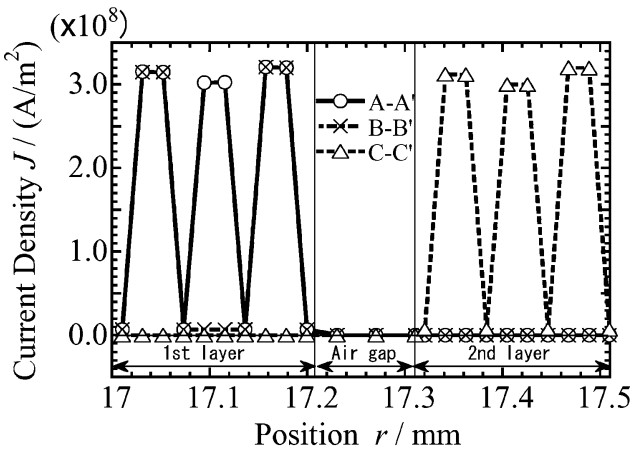

(b)

Fig. 5. Analysis results at $I=3.8 \mathrm{kA}, T=77.3 \mathrm{~K}$. (a) Distribution of magnetic flux density. (b) Distribution of current density.

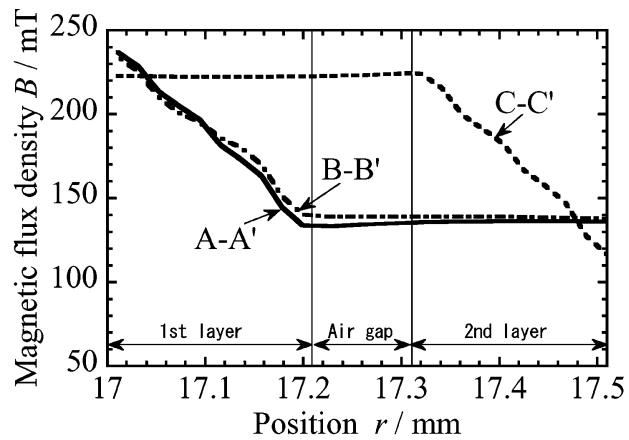

(a)

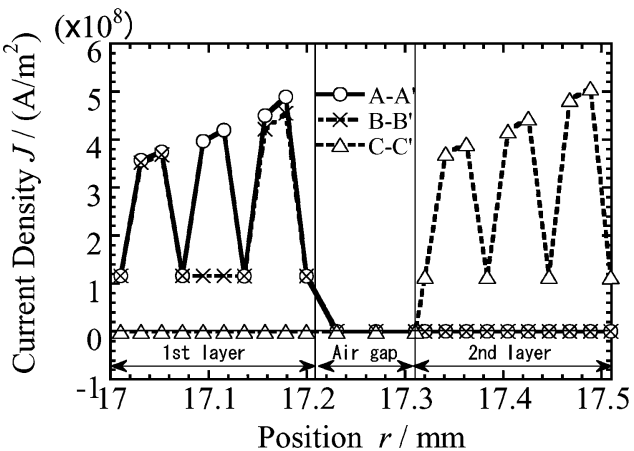

(b)

Fig. 6. Analysis results at $I=19 \mathrm{kA}, T=77.3 \mathrm{~K}$. (a) Distribution of magnetic flux density. (b) Distribution of current density.

one layer. Further, the distribution of current density in the 1 st and 2nd layer is almost consistent (Fig. 5(b)), because $B_{\text {eq }}$ is less than $B_{\text {self }}$. That is, the critical current property has no magnetic field dependence for this value of conducting current. On the other hand, analysis results for $I=19 \mathrm{kA}$ is also shown in Fig. 6. This value is considered to be fault current and much
TABLE I

COMPARISON OF AC LOSSES AT DIFFERENT CONDUCTING CURRENT VALUES $(T=77.3 \mathrm{~K})$

\begin{tabular}{lcc}
\hline \hline & AC loss $(\mathrm{W} / \mathrm{m})$ & \\
& $I=3.8 \mathrm{kA}$ & $I=19 \mathrm{kA}$ \\
\hline$W_{1, \mathrm{Bi}}$ & 0.53 & 21.7 \\
$W_{1, \mathrm{Ag}}$ & 0.02 & 19.1 \\
$W_{1, \text { total }}$ & 0.55 & 40.8 \\
\hline$W_{2, \mathrm{Bi}}$ & 0.53 & 21.7 \\
$W_{2, \mathrm{Ag}}$ & 0.02 & 18.5 \\
$W_{2, \text { total }}$ & 0.55 & 40.2 \\
\hline$W_{\text {total }}$ & 1.10 & 81.0 \\
\hline
\end{tabular}

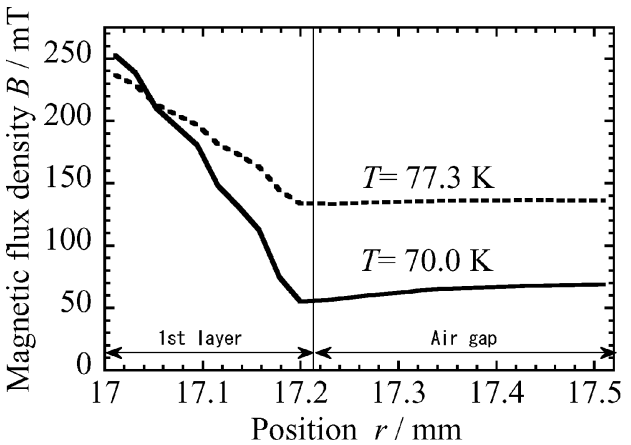

(a)

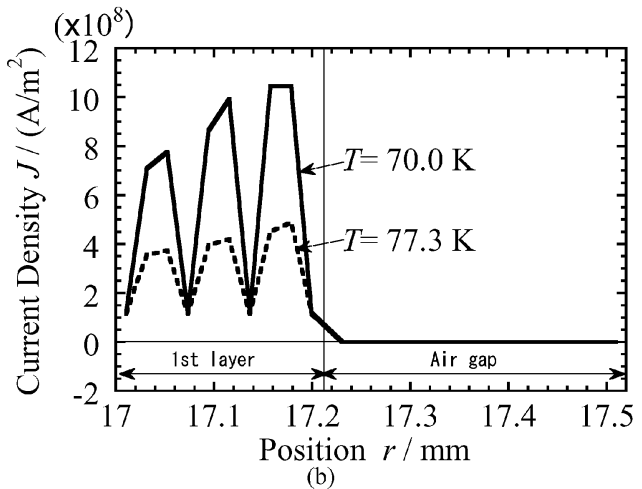

Fig. 7. Analysis results for different operating temperatures at $I=19 \mathrm{kA}$. (a) Distribution of magnetic flux density. (b) Distribution of current density.

flux is leaked outside. As can be seen in (b), the current density is decreased by reducing $r$ due to the magnetic field dependency as well as magnetic anisotropy of $J-E$ curves. That is, $B_{\mathrm{eq}}$ becomes greater than $B_{\text {self }}$ for this current value (see Fig. 1).

Table I shows the calculation results of $\mathrm{AC}$ losses $(W)$ for different conducting current values at $T=77.3 \mathrm{~K}$. Here, the first index denotes the layer number (1: first layer, 2: second layer), and the second means the calculation region (Bi: $\mathrm{Bi}-2223$ superconducting area, $\mathrm{Ag}$ : silver area, total: $\mathrm{Bi}+\mathrm{Ag}$ ). As can be seen, the $\mathrm{AC}$ loss for $\mathrm{Bi}-2223$ region, e.g., $W_{1, \mathrm{Bi}}$, is more than one order greater than that for silver area, e.g., $W_{1, \mathrm{Ag}}$, at $I=3.8 \mathrm{kA}$. This is because a large shielding current flows in the Bi-2223 filaments. On the other hand, AC loss of Bi-2223 filaments is comparable to that of silver sheath due to the large current sharing at $I=19 \mathrm{kA}$.

Total loss $\left(W_{\text {total }}\right)$ is enlarged enormously by increasing conducting current from $3.8 \mathrm{kA}$ to $19 \mathrm{kA}$. Namely, increment of conducting current at 5 times results in enlargement of AC loss at 80 times, and this result is ascribed to the nonlinear current transport properties of $\mathrm{Bi}-2223$ superconducting tape. 
TABLE II

COMPARISON OF AC LOSSES AT DIFFERENT TEMPERATURES $(I=19 \mathrm{kA})$

\begin{tabular}{lcc}
\hline \hline & AC loss $(\mathrm{W} / \mathrm{m})$ & \\
& $T=77.3 \mathrm{~K}$ & $T=70.0 \mathrm{~K}$ \\
\hline$W_{1, \mathrm{Bi}}$ & 21.7 & 23.0 \\
$W_{1, \mathrm{Ag}}$ & 19.1 & 8.0 \\
$W_{1, \text { total }}$ & 40.8 & 31.0 \\
\hline$W_{2, \mathrm{Bi}}$ & 21.7 & 23.3 \\
$W_{2, \mathrm{Ag}}$ & 18.5 & 7.7 \\
$W_{2, \text { total }}$ & 40.2 & 31.0 \\
\hline$W_{\text {total }}$ & 81.0 & 62.0 \\
\hline \hline
\end{tabular}

\section{B. Shielding Performance for Different Operating}

Temperatures $(\mathrm{I}=19 \mathrm{kA})$

The shielding performance is also analyzed for different operating temperatures, i.e., $77.3 \mathrm{~K}$ and $70.0 \mathrm{~K}$. The temperature at $70.0 \mathrm{~K}$ is regarded as the subcooled condition. In this subsection, the conducting current at $19 \mathrm{kA}$ is considered. Fig. 7 shows the analysis results. The distribution of magnetic flux density, as well as current density, is plotted along A- $\mathrm{A}^{\prime}$ (see Fig. 3 for $\left.\mathrm{A}-\mathrm{A}^{\prime}\right)$. As can be easily seen, the shielding characteristics is improved by decreasing the temperature from $77.3 \mathrm{~K}$ to $70.0 \mathrm{~K}$, i.e., the leakage flux is reduced to be half at $r=17.5 \mathrm{~mm}$ (Fig. 7(a)). This is surely because of the improvement of current transport capability. Therefore, the current density flowing in the Bi-2223 filaments is enlarged at $70.0 \mathrm{~K}$ (Fig. 7(b)).

Table II shows the comparison of AC losses. It can be seen that the $\mathrm{AC}$ losses in Bi-2223 filaments, e.g., $W_{1, \mathrm{Bi}}$, are enlarged by decreasing the operating temperature, and then that in silver sheath, e.g., $W_{1, \mathrm{Ag}}$, is reduced correspondingly. Namely, the current sharing to the silver sheath is suppressed by decreasing the operating temperature, because of the improvement of $J-$ $E$ property in Bi-2223 filaments. Therefore, the resultant total $\left(W_{\text {total }}\right)$ loss is reduced.

\section{CONCLUSION}

The analysis code of the shielding layers in HTS cable was developed taking account of the multifilamentary structure for $\mathrm{Bi}-2223 / \mathrm{Ag}$ tape and the spiral structure for the conductor. The $J-E$ constitutive relation, which can express quantitatively the dependencies of temperature, magnetic field intensity and its ap- plied direction, was introduced for 3-dimensional finite element method. As demonstrations, the shielding performances for different conducting currents and operating temperatures were investigated. It should be noted that the above mentioned characteristics can be estimated quantitatively and systematically by using our analysis code.

However, the analysis code that includes not only the shielding layers but also the conducting layers has to be developed for the exact estimation of the cable performance and the optimal design of the system, and then this will be our next target.

\section{REFERENCES}

[1] N. Steve, M. Nassi, M. Bechis, P. Ladiè, N. Kelley, and C. Wakefield, "High temperature superconducting cable field demonstration at Detroit Edison,” Physica C, vol. 354, pp. 49-54, 2001.

[2] T. Masuda, T. Kato, H. Yumura, M. Watanabe, Y. Ashibe, K. Ohkura, C. Suzawa, M. Hirose, S. Isojima, K. Matsuo, S. Honjo, T. Mimura, T. Kuramochi, Y. Takahashi, H. Suzuki, and T. Okamoto, "Verification tests of a $66 \mathrm{kV}$ HTSC cable system for practical use (first cooling tests)," Physica C, vol. 378-381, pp. 1174-1180, 2002.

[3] S. Honjo, M. Shimodate, Y. Takahashi, T. Masuda, H. Yumura, C. Suzawa, S. Isojima, and H. Suzuki, "Electric properties of a $66 \mathrm{kV}$ 3-core superconducting power cable system," IEEE Trans. Appl. Supercond., vol. 13, no. 2, pp. 1952-1955, 2003.

[4] O. Tonnesen, M. Däumling, K. H. Jensen, S. Kvorning, S. K. Olsen, C. Traholt, E. Veje, D. Willén, and J. Ostergaard, "Operation experiences with a $30 \mathrm{kV} / 100 \mathrm{MVA}$ high temperature superconducting cable system,” Supercond. Sci. Technol., vol. 17, no. 2, pp. S101-S105, 2004.

[5] Y. Xin, B. Hou, Y. Bi, K. Cao, Y. Zhang, S. Wu, H. Ding, G. Wang, Q. Liu, and Z. Han, "China's 30 m, 35 kV/2 kA AC HTS power cable project," Supercond. Sci. Technol., vol. 17, no. 4, pp. S332-S335, 2004.

[6] K. Ueda, O. Tsukamoto, S. Nagaya, H. Kimura, and S. Akita, "R\&D of a $500 \mathrm{~m}$ superconducting cable in Japan," IEEE Trans. Appl. Supercond., vol. 13, no. 2, pp. 1946-1951, 2003.

[7] K. Yamafuji and T. Kiss, "A new interpretation of the glass-liquid transition of pinned fluxoids in high- $T_{\mathrm{c}}$ superconductors," Physica $C$, vol. 258, pp. 197-212, 1996.

[8] T. Kiss, T. Nakamura, M. Inoue, M. Takeo, F. Irie, and K. Yamafuji, "Transport characteristics in high $T_{\mathrm{c}}$ superconductors," in Proc. 15th Int. Conf. Magnet Technol., 1998, pp. 1052-1055.

[9] T. Nakamura, S. Tsuchiya, A. Fujio, T. Hoshino, I. Muta, and M. Yamaguchi, "Angular dependence of $E-J$ characteristics and dissipative properties in Bi-2223/Ag tape," Supercond. Sci. Technol., vol. 15, pp. 230-235, 2002.

[10] T. Nakamura, H. Kanzaki, T. Tsuboi, K. Higashikawa, T. Hoshino, and I. Muta, "Analysis of shielding property in Bi-2223/Ag multifilamentary tapes with multi-layer arrangement," J. Materials Process. Technol., to be published. 